\title{
Surface Plasmons on a Lossy Left-Handed Medium
}

\author{
M. Alam, J. Meier, J. S. Aitchison, and M. Mojahedi \\ Department of Electrical and Computer Engineering, \\ University of Toronto, Toronto, Ontario, Canada M5S 3G4
}

\section{Introduction}

Surface plasmons (SPs) at the interface of left and right handed materials show many unique features. For example, left handed materials (LHMs) support both transverse magnetic (P-type) and transverse electric (S-type) SPs modes and the backward propagation of a plasmonic wave. In the past few years, SPs on the LHMs have attracted some interest [1-3]. However, the effects of material losses on these modes are yet to be fully explored. Here, we study the effects of dielectric and magnetic losses on a SP mode propagating on a LHM slab embedded in a lossless right handed medium. We show that the existence of a long range SP solution depends on the type of loss present in the material. In the case of S-type SP it is found that magnetic loss precludes the existence of long range modes.

\section{Analysis and Results}

We analyze a LHM slab surrounded by semi infinite right handed material (RHM, air) as shown in Fig.1. The slab occupies the region $0<y<d$. Ruppin has shown that such a slab can support both P- and S-polarized SPs [2]. We will only consider the S-type SPs because results for the P-type can be easily deduced.

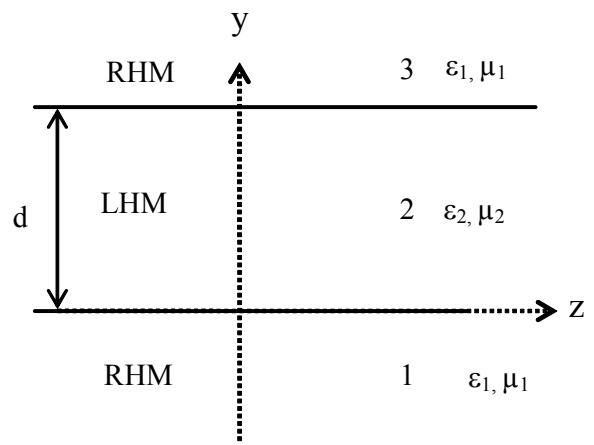

Fig.1: LHM slab surrounded by semi-infinite RHM (air).

For the S-type SP the field expressions are

$$
\begin{gathered}
\vec{E}_{1}=\hat{x} A e^{a_{0} y+i k z}, \vec{H}_{1}=-\frac{c}{\omega \mu_{1}} A\left(k \hat{y}+i a_{0} \hat{z}\right) e^{a_{0} y+i k z} \\
\vec{E}_{2}=\widehat{x}\left(B e^{-a y}+C e^{a y}\right) e^{i k z}, \vec{H}_{2}=-\frac{c}{\mu_{2} \omega}\left[B(k \hat{y}-i a \hat{z}) e^{-a y}+C(k \hat{y}+i a \hat{z}) e^{a y}\right] e^{i k z}
\end{gathered}
$$




$$
\vec{E}_{3}=\hat{x} D e^{-a_{0} y+i k z}, \vec{H}_{3}=-\frac{c}{\omega \mu_{1}} D\left(k \hat{y}-i a_{0} \hat{z}\right) e^{-a_{0} y+i k z}
$$

Here $\vec{E}_{j}$ and $\vec{H}_{j}$ are the electric and magnetic fields in the $j$-th region, $k$ is the wave vector component in the direction of propagation, $a$ and $a_{0}$ are the transverse wavevector component inside the slab and the surrounding material, respectively; and A, B, C and D are constants to be determined. In presence of loss the wave number $k$ becomes complex, i.e. $k=\beta+i \alpha$, where $\beta$ corresponds to the propagation constant of the mode and $\alpha$ is a measure of the attenuation.

When the slab is sufficiently thin, the SP modes supported by the two opposite surfaces at $y=0$ and $y=d$ couple to each other and symmetric and anti-symmetric SP modes are possible. The dispersion relations for the anti-symmetric and symmetric SP modes (with respect to $E_{x}$ ) are respectively [2]

$$
\begin{aligned}
& \mu_{2}=-\mu_{1} \frac{a}{a_{0}} \operatorname{coth}(a d / 2) \\
& \mu_{2}=-\mu_{1} \frac{a}{a_{0}} \tanh (a d / 2)
\end{aligned}
$$

Following [2], a LHM operating in the microwave region and implemented by a combination of split ring resonators (SRRs) and thin wire arrays is considered in this work. The dielectric constant of the thin wire array is given by [4]

$$
\varepsilon(f)=1-\frac{f_{p}^{2}}{f\left(f+i \gamma f_{p}\right)},
$$

where $f_{p}$ is the plasma frequency and $\gamma$ is the electric damping coefficient. The magnetic permeability of the SRR can be expressed as [5]

$$
\mu(f)=1-\frac{F f^{2}}{f^{2}-f_{0}^{2}+i \Gamma f} .
$$

Here, $f_{0}$ is the resonance frequency and $\Gamma$ is the magnetic damping coefficient.

As in [2], $f_{p}, f_{0}$ and $F$ are chosen to be $10 \mathrm{GHz}, 4 \mathrm{GHz}$ and 0.56 , respectively. For these values the medium acts as a LHM for frequencies between 4 to $6 \mathrm{GHz}$. In the case of a lossless LHM slab the propagation constant $\beta$ decreases with increasing slab thickness for both symmetric and anti-symmetric modes as shown in Fig. 2. This is contrary to the case of RHM slab. For a RHM slab, $\beta$ increases with increasing slab thickness for the symmetric mode and decreases for the anti-symmetric mode [6].

The effects of loss are considered next. Following [4] and [5], $\gamma=0.1$ and $\Gamma=0.3 \mathrm{GHz}$ are chosen. The dispersion relations for the two modes in the presence of loss are shown in Fig. 3. The attenuation is very high compared to the case of RHM. For example at 193.5 $\mathrm{THz}(1.55 \mu \mathrm{m}$ wavelength) for a $40 \mathrm{~nm}$ thick silver film surrounded by air, the propagation length is about 130 times the free space wavelength. On the contrary, for the 
LHM under study, the propagation distance for the SP is about one free space wavelength or less over the frequency range where the material is a LHM.
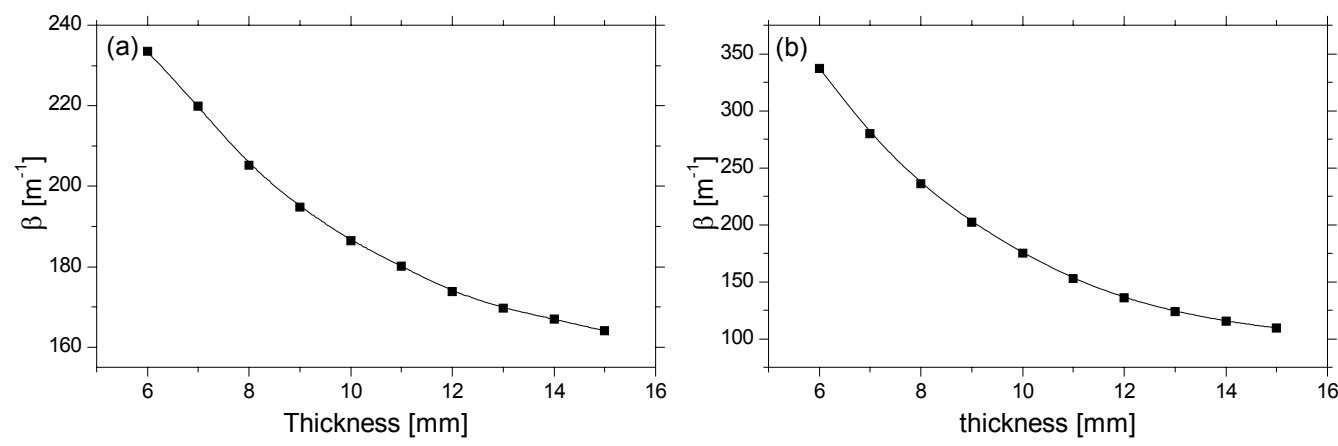

Fig. 2: Variation of the wavevector $\beta$ for (a) symmetric branch at $5 \mathrm{GHz}$, (b) anti-symmetric branch at $4.6 \mathrm{GHz}$
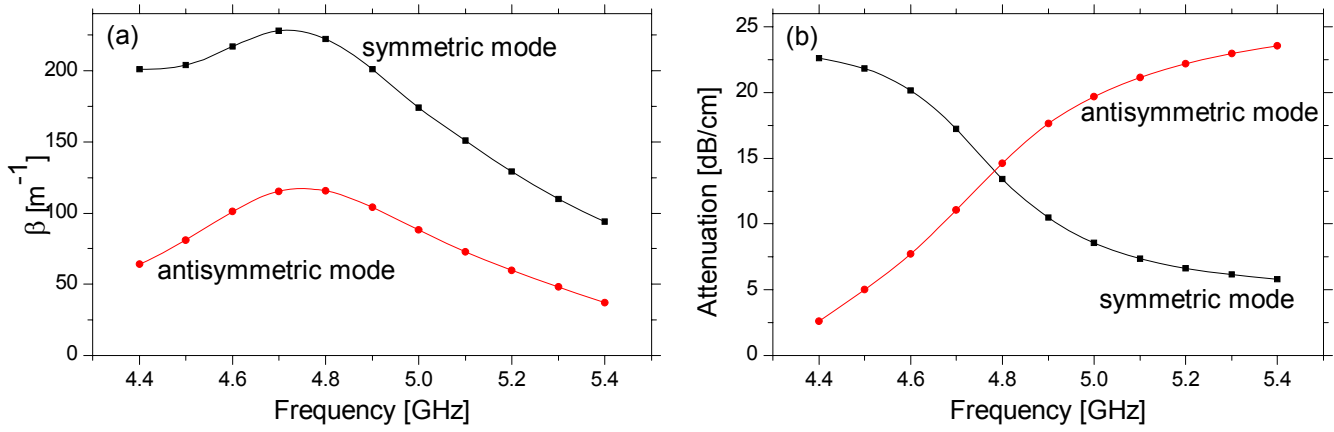

Fig. 3: Dispersion relations for the two SP modes for a $10 \mathrm{~mm}$ thick LHM slab:

(a) propagation constant; (b) attenuation

The usual approach for reducing the attenuation of SPs in the optical regime is to reduce the slab thickness. For a metal film surrounded by lossless dielectric media, when the film thickness is reduced, the symmetric mode suffers less attenuation [6]. We examine the applicability of the same technique for the LHM slab. While in LHM both dielectric and magnetic losses are present, we scrutinize the role of the two loss mechanisms separately. Two LHM slabs are considered, one having only dielectric loss $(\gamma=0.1 \mathrm{GHz}$ and $\Gamma=0 \mathrm{GHz}$ ), and the other having only magnetic loss ( $\gamma=0 \mathrm{GHz}$ and $\Gamma=0.3 \mathrm{GHz})$. For these values of $\gamma$ and $\Gamma, \varepsilon_{2}=-2.85+\mathrm{i} 0.77, \mu_{2}=-0.55$ for the first slab, and $\varepsilon_{2}=-3$, $\mu_{2}=-0.47+\mathrm{i} 0.27$ for the second slab at the frequency of $5 \mathrm{GHz}$. Here we present only results for the symmetric mode. We found that the asymmetric mode in LHM behaves like the asymmetric mode in a RHM, where it shows increased attenuation with reduced slab thickness. Figure 4(a) shows that when only dielectric loss is present, the attenuation is reduced with decreasing slab thickness. However, in the presence of magnetic loss the attenuation increases for thinner slabs. As a result, realizing a low loss LHM waveguide for SP requires a reduction of the loss beyond the values found here. 
For the S-type SP on a lossless LHM slab, the $H_{y}$ and $E_{x}$ components are in phase and $H_{z}$ is out of phase with them. The power flow is then exclusively in the $z$-direction. In the presence of loss, the phase relations are changed, and the $E_{x}$ and $H_{z}$ are no longer completely out of phase. As a result, the power flow has a $y$-component, i.e. the SP loses power to radiation. This effect can be very significant in case of LHM when $\mu$ is complex. To assess this we define a factor $\eta=P_{y} / P_{z}$. Here $P_{y}$ is the total power flow in the $y$-direction and $P_{z}$ is the total power flow in the $z$-direction. Fig. 4(b) shows the variation of $\eta$ for the two cases. When only $\mu$ is complex, a significant amount of power is lost because of radiation. The effect is less severe when only $\varepsilon$ is complex.
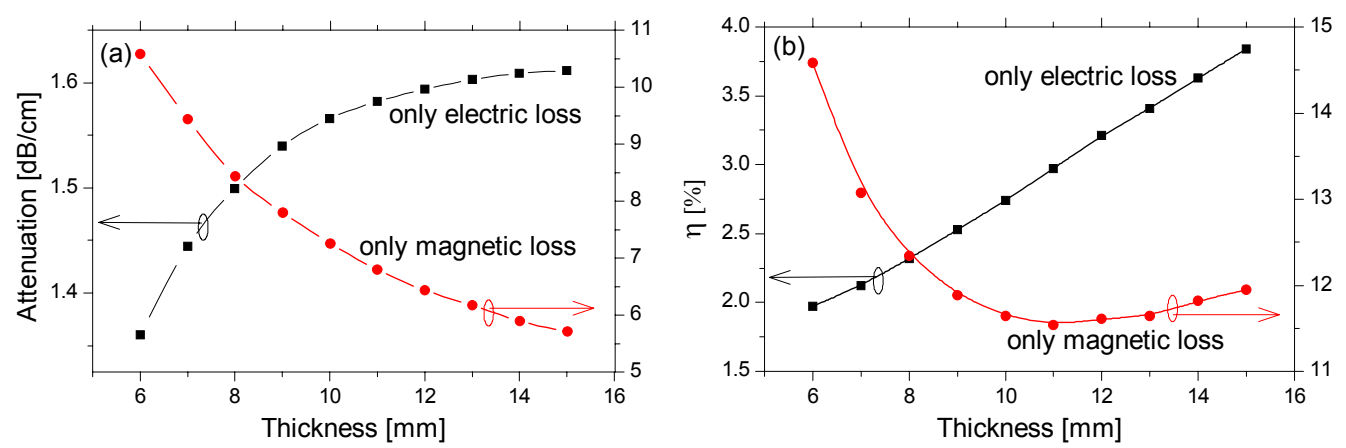

Fig. 4: (a) Variation of the attenuation with thickness. (b) Variation of the ratio of radiated power $\left(P_{y}\right)$ to guided power $\left(P_{z}\right)$ with varying slab thickness. The frequency is $5 \mathrm{GHz}$ for both figures and slabs are left-handed.

\section{Conclusion}

It is shown that SPs propagating on a LHM slab suffer significantly more attenuation in the presence of loss compared to the SPs on RHM. The attenuation critically depends on the loss mechanism. These results can be useful in designing plasmonic devices based on LHM for practical applications.

\section{References}

[1] R. Ruppin, "Surface polaritrons on left-handed medium", Phys. Lett. A, 277, 61-64 (2000).

[2] R. Ruppin, "Surface polaritrons on a left-handed material slab", J. Phys.: Condens. Matter, 13, 1811-1818 (2001).

[3] K. Park, B. J. Lee, C. Fu, Z. M. Zhang, "Study of the surface and bulk polaritrons with a negative index metamaterial", J. Opt. Soc. Am B, 22, 1016-1023 (2005).

[4] J. B. Pendry, A. J. Holden, W. J. Stewart, "Extremely low frequency plasmons on metallic mesostructures", Phys. Rev. Lett., 76, 4773-4776 (1996).

[5] J. B. Pendry, A. J. Holden, W. J. Stewart, "Magnetism from conductors and enhanced nonlinear phenomenon", IEEE T. Microw. Theory, 47, 2075-2083 (1999).

[6] J. J. Burke, G. I. Stegeman, "Surface-polaritron like waves guided by thin, lossy metal films”, Phys. Rev. B, 33, 5855-5567 (1986). 